\title{
What is the scale of
} prescribing errors committed

\section{by junior doctors? A systematic review}

\section{Sarah Ross, ${ }^{1}$ Christine Bond, ${ }^{2}$ Helen Rothnie, ${ }^{2}$ Sian Thomas $^{2} \&$ Mary Joan Macleod ${ }^{1}$}

Departments of ${ }^{1}$ Medicine and Therapeutics and ${ }^{2}$ General Practice and Primary Care, University of Aberdeen, Aberdeen, UK

\section{Correspondence}

Dr Sarah Ross, Department of Medicine and Therapeutics, University of Aberdeen, Foresterhill, Aberdeen AB25 2ZN, UK. Tel.: + 4401224553015

Fax: + 4401224554761

E-mail:s.ross@abdn.ac.uk

Keywords

drug prescription, medication error physicians

Received

18 July 2008

Accepted

7 October 2008

Published Early View

16 December 2008

\section{WHAT IS ALREADY KNOWN ABOUT}

\section{THIS SUBJECT}

- Prescribing errors account for a substantial proportion of medication errors and cause the most significant problems.

- There is a dearth of accurate information on the prevalence of prescribing errors, with estimates from 1 to $100 \%$ of all patients admitted to hospital.

\section{WHAT THIS STUDY ADDS}

- This review reports the wide ranges of error rates seen, which cannot be compared due to differences in methodology and error definitions used.

- A well-conducted study of prescribing errors by junior doctors using standard definitions and methodology is urgently needed to allow development and assessment of appropriate interventions.
AIMS

Prescribing errors are an important cause of patient safety incidents, generally considered to be made more frequently by junior doctors, prevalence and causality are unclear. In order to inform the design of an educational intervention, a systematic review of the literature on prescribing errors made by junior doctors was undertaken.

\section{METHODS}

Searches were undertaken using the following databases: MEDLINE; EMBASE; Science and Social Sciences Citation Index; CINAHL; Health Management Information Consortium; PsychINFO; ISI Proceedings; The Proceedings of the British Pharmacological Society; Cochrane Library; National Research Register; Current Controlled Trials; and Index to Theses. Studies were selected if they reported prescribing errors English, published since 1990 and undertaken in Western Europe, North America or Australasia.

\section{RESULTS}

Twenty-four studies meeting the inclusion criteria were identified. The range of error rates was $2-514$ per 1000 items prescribed and 4.2-82\% of patients or charts reviewed. Considerable variation was seen in design, methods, error definitions and error rates reported.

\section{CONCLUSIONS}

The review reveals a widespread problem that does not appear to be associated with different training models, healthcare systems or infrastructure. There was a range of designs, methods, error definitions and error rates, making meaningful conclusions difficult. No definitive study of prescribing errors has yet been conducted, and is urgently needed to provide reliable baseline data for interventions aimed at reducing errors. It is vital that future research is well constructed and generalizable using standard definitions and methods. 


\section{Introduction}

Medication errors are the second most common cause of patient safety incidents [1], with prescribing errors an important component of these [2]. Using Human Error Theory [3], errors can be divided into individual and systems factors. Although much of the current emphasis in patient safety is on systems factors, individual doctors' actions are also important. In a study of prescribing errors using Human Error Theory, Dean et al. [4] reported that $43 \%$ of errors were mistakes or violations, whereas $57 \%$ were lapses. This suggests that knowledge-based errors are important and could potentially be addressed by educational interventions at undergraduate or postgraduate level. Focusing interventions on medical students and junior doctors is appropriate for two reasons. First, junior doctors are responsible for the majority of actual prescribing in hospitals (having been reported as responsible for $91 \%$ of prescribing errors [4]), although they may not be responsible for all prescribing decisions. Second, training at these stages may be more effective and efficient than at a later point in a doctor's career. In order to design interventions, it is important to understand the nature of individual factors and the size of the problem. However, there is a dearth of consistent information on the prevalence of prescribing errors. Prescribing errors are reported to affect $1-100 \%$ of all patients admitted to hospital [5].

In order to clarify the situation, a systematic review of the current published evidence to answer the research question 'how many prescribing errors are committed by junior doctors' was undertaken.

\section{Methods}

We included studies of any experimental design that addressed prescribing errors, involved junior doctors, were published since 1990 in English, from the UK, Europe, North America, Australia or New Zealand, and were conducted in primary or secondary care. Junior doctors are defined as foundation doctors, junior house officers, senior house officers or registrars in the UK and interns, residents or fellows in other countries.

The search strategy included the terms prescription, prescribing, junior doctors and error (see Appendix 1). Searches were undertaken in July 2007 using the following databases: MEDLINE; EMBASE; Science and Social Sciences Citation Index; Cumulative Index to Nursing and Allied Health Literature (CINAHL); Health Management Information Consortium; PsychINFO; ISI Proceedings; The Proceedings of the British Pharmacological Society; Cochrane Library; National Research Register; Current Controlled Trials; Index to Theses; and Internet via Google. Subsequent automatic alerts were generated. In addition, hand searching of the reference lists from included studies was carried out by one researcher (S.R.).
Citation titles retrieved in the search were each independently reviewed against the inclusion criteria by two researchers (S.R. and M.J.M. or C.B.). Abstracts associated with included titles were then retrieved and again each reviewed by two researchers (S.R. and C.B.). Finally, full papers for potentially eligible studies were retrieved and independently reviewed by two researchers (H.R. and S.T.). Disagreements were resolved by discussion.

Data abstraction from full papers was undertaken independently by two researchers (H.R. and S.T.) with differences resolved by consensus with a third researcher (S.R.). A form, based on the Scottish Intercollegiate Guidelines Network [6] and the Critical Appraisal Skills Programme [7], was developed to support consistent abstraction of data to answer the research question. It contained the following information: study design, study aim, participants, setting, possible confounding, methods of data collection, blinding, statistical analysis, definitions used and outcomes. The form was piloted, refined and finalized by consensus.

The methodological quality of the studies was subjectively rated independently by S.T. and H.R. using a modified version of the Effective Public Health Practice Project Quality Assessment Tool for Quantitative Studies [8]. The following aspects were assessed: study aim, participant selection, control of confounding, data collection, assessor blinding, participant blinding, statistical analysis and withdrawals. A summary grade - strong, moderate or weak was then assigned to each criterion. Disagreements were resolved by discussion.

It was anticipated that the selected studies would not be amenable to meta-analysis, and narrative qualitative analysis was planned. Where possible, published data were computed to a 'common unit of measurement'.

\section{Results}

Initial searching identified 2091 studies. A further 28 were added from MEDLINE/EMBASE alerts and five from handsearching of references, giving a total of 2124. A flowchart illustrating the progressive study selection and numbers at each stage is shown (Figure 1). Five papers, not available via interlibrary loan within the UK, were excluded. Twentyfour studies met the inclusion criteria.

Eight studies were undertaken in the UK, 10 in the USA, four in Canada and one each in Australia and the Netherlands. The majority were conducted in a hospital environment.

The majority of studies were observational; two were described as randomized controlled trials but were judged to be observational studies. Eighteen studies were prospective and six retrospective.

Seven papers were categorized as methodologically strong with regard to study aim, none in selection of participants, three for controlling confounding, five for data collection, four for blinding of assessors, five for blinding 


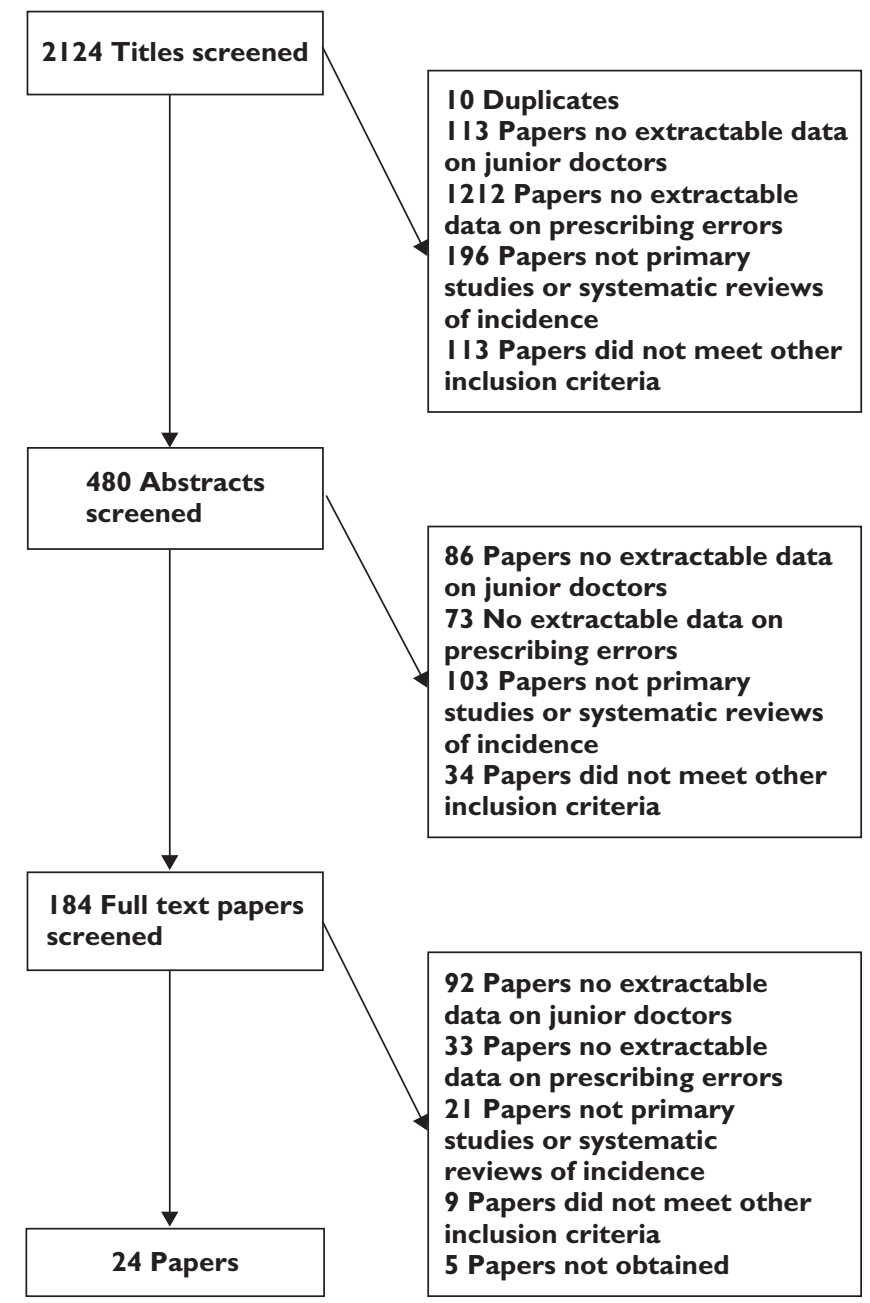

Figure 1

Flowchart of included studies

of participants, three for statistical analysis and seven for withdrawals (see Table 1).

A number of specific issues were identified that limit comparisons. First, there was no consistent definition of error across studies, with different combinations of 35 possible criteria being used. Of these, wrong dose was the most commonly used (15 studies), nine criteria were only used by two studies and 12 by only one study. Table 2 shows an abbreviated list of possible criteria used in defining prescribing errors. These could be grouped into those that are a potential danger to the patient (wrong drug, dose, allergy), those that may be a danger or merely require clarification (e.g. some interactions, or medicines causing computer alerts), and those that increase the cost of the medicine (e.g. brand name use, nonformulary items).

Second, data collection methods varied between studies, leading to possible biases and different error rates. Units for reporting of error rates or numbers varied, but could be roughly grouped into three types: errors made by junior doctors per single medication item $(n=11)$; errors made by junior doctors per whole drug chart (multiple medications) or per patient ( $n=9)$; and absolute numbers of errors made by junior doctors $(n=4)$. Even within these groups, there were inconsistencies. For example, those reporting per item rates report either for items written by the junior doctors or all items prescribed by any doctor. One study reported the percentage of errors made by juniors of all errors made by any doctor. For these reasons, the data are presented by individual study expanding on each issue, with a summary of the findings reported in Table 3.

\section{Studies reporting errors per item}

Lesar [9] quantified prescribing errors made by physicians in a US teaching hospital, to determine associated risk factors and to assess the risk to patients from errors. All orders written were reviewed by centralized staff pharmacists prior to dispensing and potential errors identified in these were then discussed with prescribing staff. Of 289411 orders, 905 errors were reported (3.13 per 1000 orders). Junior staff made 864 errors (95.5\%), giving a calculated error rate of three errors made by junior staff per 1000 items prescribed by all doctors.

Bordun [10] studied prescribing errors in a single paediatric intensive care unit in Australia and evaluated the incidence, type and significance of prescribing errors. The Intensive Care Unit ward pharmacist reviewed every medication order and noted errors, which were then agreed and categorized by a consultant physician. During the study period, 68 of 1518 medication orders were judged to be errors ( 45 per 1000). Only 53 errors could be traced to the prescriber, of which 46 (87\%) were made by junior staff. After adjustment for those errors not accounted for, this gives a calculated error rate of 38 errors made by junior staff per 1000 items prescribed by all doctors.

Ho [11] characterized the number, frequency, origin and outcome of prescribing errors in a Canadian tertiary care teaching hospital. There were 1330 prescribing errors over a 25-week period. Residents were responsible for 479 errors (36\%). In the study period, 237798 items were prescribed, giving an overall rate of five errors per 1000 items. Residents had a reported error rate of two per 1000 items prescribed by all doctors.

Howell [12] studied the effect of an educational intervention for residents in a US family care centre. At baseline, 23 errors were identified in 128 medication orders and 34 out of 172 post intervention. This is reported as 179 per 1000 items written by juniors pre-intervention and 197 per 1000 items written by juniors post intervention.

Bizovi [13] determined whether the introduction of computer-aided prescribing reduced prescribing errors in a US emergency department. Pre-intervention there were 54 errors in 2326 medication items ordered by any doctor (reported as 23 errors per 1000 items) and 32 errors in 1280 items made by residents, which calculates as 25 errors per 1000 items written by juniors. Post intervention, 11 errors 


\section{Table 1}

Overall methodological quality of included studies

\begin{tabular}{|lcccccccc|}
\hline $\begin{array}{l}\text { Overall grade } \\
\boldsymbol{n}(\%)\end{array}$ & Study aim & $\begin{array}{l}\text { Selection of } \\
\text { participants }\end{array}$ & $\begin{array}{l}\text { Control of } \\
\text { confounding }\end{array}$ & $\begin{array}{l}\text { Data } \\
\text { collection }\end{array}$ & $\begin{array}{l}\text { Blinding of } \\
\text { assessors }\end{array}$ & $\begin{array}{l}\text { Blinding of } \\
\text { participants }\end{array}$ & $\begin{array}{l}\text { Statistical } \\
\text { analysis }\end{array}$ & Withdrawals \\
\hline Strong & $7(29)$ & 0 & $3(13)$ & $5(21)$ & $4(17)$ & $5(21)$ & $3(13)$ & $7(29)$ \\
Moderate & $16(67)$ & $19(79)$ & $7(29)$ & $14(58)$ & $15(62)$ & $7(29)$ & $6(25)$ & 0 \\
Weak & $1(4)$ & $5(21)$ & $14(58)$ & $5(21)$ & $5(21)$ & $12(50)$ & $15(62)$ & $17(71)$ \\
\hline
\end{tabular}

\section{Table 2}

Error definitions

\begin{tabular}{|lc|}
\hline Criteria & Number of studies using \\
\hline Wrong dose & 15 \\
\hline Wrong frequency & 14 \\
Omitted information & 11 \\
\hline Wrong route & 8 \\
\hline Contraindicated due to allergy & 6 \\
\hline Wrong drug & 6 \\
\hline Inaccurate information & 5 \\
\hline Other contraindication & 5 \\
\hline Definition from Dean [32] & 4 \\
\hline Illegibility & 4 \\
\hline Interaction & 4 \\
\hline Unclear quantity & 4 \\
\hline Illegality & 3 \\
\hline Wrong patient & 3 \\
\hline
\end{tabular}

in 1594 items were made by any doctor (reported as seven per 1000), and six in 1114 were made by residents (calculated as five errors made by junior staff per 1000 items prescribed by all doctors).

Dean [14] studied the incidence, type and significance of prescribing errors in a UK teaching hospital. In 36168 orders, there were 538 errors. Of these errors, the prescriber could be identified in 482, of which 472 errors (98\%) were made by junior staff (calculated rate is 15 errors per 1000 total items).

Fijn [15] explored an epidemiological framework to assess predictors of prescribing errors in two teaching hospitals in the Netherlands. Data collection included all new prescriptions over a 14-day period. There were 449 errors in 1913 prescription items written by any doctor (assumed as not explicit in paper), giving a rate of 235 errors per 1000 items. Junior doctors were 1.57 times more likely to cause an error than senior staff (calculated as 143 errors made by junior staff per 1000 items prescribed by all doctors).

Anton [16] studied a computerized physician order entry system in a UK renal medicine ward. Alerts generated by the system were used as a proxy measure for potential errors. Data were collected from the computer system for a 2-month period. Of 5995 prescription items ordered, 5136 were made by junior doctors. There were 6592 alerts, of which junior doctors received 5462 warning alerts. Of these warnings, 2820 were ignored, suggesting that 2642 warnings reflected true errors (calculated as 514 errors made by junior staff per 1000 items prescribed by juniors).

Hendey [17] compared the error rates of residents in a community-based teaching hospital in the USA, between on call or post on call junior staff. Errors were logged by the pharmacy department, then a retrospective chart review was undertaken by researchers. There were 177 errors in 8195 medication orders (reported as 22 errors made by junior staff per 1000 items prescribed by juniors).

Stubbs [18] examined the nature, frequency and severity of prescribing errors in nine UK psychiatric hospitals. There were 880 errors in 22036 items; however, 357 did not meet the inclusion criteria (e.g. errors relating to the whole chart such as missing information, or which did not fit the definition but were off formulary, off license or other errors). Nonconsultant staff made 329 of the 523 errors (63\%), calculated as a rate of 15 errors made by junior staff per 1000 items prescribed by all doctors.

Walsh [19] determined the frequency and types of medication errors attributable to a computerized physician order entry system in a US study of a paediatric intensive care unit. There were 104 errors in 6916 medication orders made by residents for 352 admissions, calculated as 15 medication errors per 1000 total items. No breakdown of errors is given, therefore prescribing error data cannot be extracted. However, the paper does report that $71 \%$ of the 71 serious errors were at the drug ordering (prescribing) stage.

\section{Studies reporting error per patient or drug chart}

Shaughnessy [20] tested whether educational intervention improved the prescription writing skills of residents in a US family medicine centre. Data were collected from residents over three 4-monthly periods by pharmacy staff, pre, during and post intervention; $21 \%$ of charts contained an error prior to the intervention and $17 \%$ after the intervention.

Shaughnessy [21] also conducted a further study in the same setting, over a longer time frame. At baseline $14.4 \%$ of drug charts contained an error, with $6 \%$ post intervention. 
Table 3

Summary of included primary studies

\begin{tabular}{|c|c|c|c|c|c|}
\hline Study & Study design & Setting and subjects & Error definition & Intervention (if applicable) & Main findings \\
\hline $\begin{array}{l}\text { Lesar et al. } \\
\qquad(1990)[9]\end{array}$ & $\begin{array}{l}\text { Prospective } \\
\text { observational }\end{array}$ & $\begin{array}{l}\text { Setting: } 640 \text {-bed tertiary care } \\
\text { teaching hospital (secondary } \\
\text { care) } \\
\text { Subjects: Adult and paediatric } \\
\text { patients ( } 21464 \text { admissions } \\
\text { during study period; } \\
\text { Physicians ( } n=840 \text {, of which } 378 \\
\text { house staff and fellows) }\end{array}$ & $\begin{array}{l}\text { Incorrect patient, drug, dosage, } \\
\text { frequency, form, route, } \\
\text { inappropriate or redundant } \\
\text { indication, contraindicated } \\
\text { medications (including } \\
\text { allergies), or orders with critical } \\
\text { information missing }\end{array}$ & None & $\begin{array}{l}905 \text { errors out of } 289411 \text { orders } \\
\text { were identified ( } 3.1 \text { per } 1000 \\
\text { items). } \\
864 \text { errors were made by junior } \\
\text { staff ( } 95.7 \% \text { ), giving a } \\
\text { calculated error rate of } 3 \text { errors } \\
\text { made by junior staff per } 1000 \\
\text { items prescribed by all doctors }\end{array}$ \\
\hline $\begin{array}{l}\text { Ho et al. (1992) } \\
\text { [11] }\end{array}$ & $\begin{array}{l}\text { Prospective } \\
\text { observational }\end{array}$ & $\begin{array}{l}\text { Setting: } 580 \text {-bed tertiary care } \\
\text { teaching hospital } \\
\text { Subjects: patients and doctors } \\
\text { and in this hospital ( } n=\text { not } \\
\text { reported). }\end{array}$ & $\begin{array}{l}\text { Any order that contained an } \\
\text { inaccuracy or omission, or } \\
\text { commenced potentially } \\
\text { detrimental therapy and was } \\
\text { captured as error or discussed } \\
\text { and changed with the } \\
\text { prescriber. Errors already } \\
\text { detected and addressed by the } \\
\text { ward pharmacy staff were not } \\
\text { included in the study }\end{array}$ & None & $\begin{array}{l}\text { In the study period, } 237798 \\
\text { items were prescribed, giving } \\
\text { an overall rate of } 5 \text { errors per } \\
1000 \text { items. Residents had a } \\
\text { reported error rate of } 2 \text { per } \\
1000 \text { items prescribed by all } \\
\text { doctors }\end{array}$ \\
\hline $\begin{array}{l}\text { Howell and } \\
\text { Jones (1993) } \\
\text { [12] }\end{array}$ & $\begin{array}{l}\text { Prospective } \\
\text { observational }\end{array}$ & $\begin{array}{l}\text { Setting: family practice centre } \\
\text { (primary care) } \\
\text { Subjects: patients ( } n=\text { not } \\
\text { reported) at a family practice } \\
\text { centre; } \\
\text { 1st year family practice residents } \\
\quad(n=8)\end{array}$ & $\begin{array}{l}\text { Omitted prescription information, } \\
\text { wrong dose or frequency, } \\
\text { unclear quantity or directions, } \\
\text { a prescription for a } \\
\text { nonprescription product or } \\
\text { failure to comply with } \\
\text { legislation }\end{array}$ & $\begin{array}{l}\text { In-service training was } \\
\text { provided to all 1st year } \\
\text { residents in prescription } \\
\text { writing and to review } \\
\text { common prescription } \\
\text { writing errors }\end{array}$ & $\begin{array}{l}\text { At baseline, } 23 \text { errors were } \\
\text { identified in } 128 \text { medication } \\
\text { orders and } 34 \text { out of } 172 \text { post } \\
\text { intervention. This is reported as } \\
179 \text { per } 1000 \text { items written by } \\
\text { juniors pre intervention and } \\
197 \text { per } 1000 \text { items written by } \\
\text { juniors post intervention }\end{array}$ \\
\hline $\begin{array}{l}\text { Dean et al. } \\
\text { (2002) [14] }\end{array}$ & $\begin{array}{l}\text { Prospective } \\
\text { observational }\end{array}$ & $\begin{array}{l}\text { Setting: teaching hospital (single } \\
\text { centre) } \\
\text { Subjects: inpatients ( } n=459 \\
\text { hospital episodes); doctors } \\
\text { ( } n=\text { not reported) in a 550-bed } \\
\text { teaching hospital }\end{array}$ & $\begin{array}{l}\text { In accordance with Dean's Delphi } \\
\text { study of prescribing errors [32] }\end{array}$ & None & $\begin{array}{l}\text { In } 36168 \text { orders, there were } 538 \\
\text { errors. Of these errors, the } \\
\text { prescriber could be identified in } \\
482 \text {, of which } 472 \text { errors } \\
(98 \%) \text { were made by junior } \\
\text { staff (calculated rate is } 15 \\
\text { errors per } 1000 \text { total items) }\end{array}$ \\
\hline
\end{tabular}


Table 3

Continued

\begin{tabular}{|c|c|c|c|c|c|}
\hline Study & Study design & Setting and subjects & Error definition & Intervention (if applicable) & Main findings \\
\hline $\begin{array}{l}\text { Fijn et al. } \\
\text { (2002) [15] }\end{array}$ & $\begin{array}{l}\text { Retrospective } \\
\text { cross-sectional }\end{array}$ & $\begin{array}{l}\text { Setting: two teaching hospitals. } \\
\text { Subjects: patients ( } n=\text { not } \\
\text { reported) requiring a } \\
\text { prescription; clinicians (senior } \\
\text { doctors) and clinician assistants } \\
\text { (junior doctors) ( } n=\text { not } \\
\text { reported) }\end{array}$ & $\begin{array}{l}\text { Errors in dose, therapeutic errors, } \\
\text { illegible prescription, missing } \\
\text { data on date, patient or } \\
\text { prescriber, unclear drug name, } \\
\text { wrong drug, unauthorized } \\
\text { abbreviation use, or route } \\
\text { missing/inaccurate }\end{array}$ & None & $\begin{array}{l}\text { There were } 449 \text { errors in } 1913 \\
\text { prescription items written by } \\
\text { any doctor (assumed as not } \\
\text { explicit in paper), giving a rate } \\
\text { of } 235 \text { errors per } 1000 \text { items. } \\
\text { Junior doctors were } 1.57 \text { times } \\
\text { more likely to cause an error } \\
\text { than senior staff (calculated as } \\
143 \text { errors made by junior staff } \\
\text { per } 1000 \text { items prescribed by } \\
\text { all doctors) }\end{array}$ \\
\hline $\begin{array}{l}\text { Anton et al. } \\
\text { (2004) [16] }\end{array}$ & $\begin{array}{l}\text { Prospective } \\
\text { observational }\end{array}$ & $\begin{array}{l}\text { Setting: } 64 \text {-bed renal unit in a } \\
\text { teaching hospital } \\
\text { Subjects: renal patients ( } n=257) \text {; } \\
\text { doctors ( } n=42 ; 9 \text { consultants, } \\
13 \text { registrars, } 6 \text { SHOs, } 14 \\
\text { PRHOs) prescribing in the renal } \\
\text { unit }\end{array}$ & $\begin{array}{l}\text { Alerts generated by a } \\
\text { computerized prescribing } \\
\text { system were used as a proxy } \\
\text { measure for potential errors }\end{array}$ & $\begin{array}{l}\text { Routine use of a } \\
\text { computerized prescribing } \\
\text { system by doctors and } \\
\text { nurses on a renal unit }\end{array}$ & $\begin{array}{l}\text { There were } 6592 \text { alerts, of which } \\
\text { junior doctors received } 5462 \\
\text { warning alerts. Of these } \\
\text { warnings, } 2820 \text { were ignored, } \\
\text { suggesting that } 2642 \text { warnings } \\
\text { reflected true errors (calculated } \\
\text { as } 514 \text { errors made by junior } \\
\text { staff per } 1000 \text { items prescribed } \\
\text { by juniors) }\end{array}$ \\
\hline $\begin{array}{l}\text { Hendey et al. } \\
\text { (2005) [17] }\end{array}$ & $\begin{array}{l}\text { Retrospective } \\
\text { observational }\end{array}$ & $\begin{array}{l}\text { Setting: university affiliated } \\
\text { community teaching hospital } \\
\text { (secondary care) } \\
\text { Subjects: adult inpatients ( } n=\text { not } \\
\text { reported) in medical/surgical } \\
\text { wards and critical care areas; } \\
\text { doctors: }(n=57) \text { residents of } \\
\text { various grades }\end{array}$ & $\begin{array}{l}\text { Any error identified by the } \\
\text { pharmacist, excluding simple } \\
\text { clarifications that did not result } \\
\text { in a change }\end{array}$ & None & $\begin{array}{l}\text { There were } 177 \text { errors in } 8195 \\
\text { medication orders (reported as } \\
22 \text { errors made by junior staff } \\
\text { per } 1000 \text { items prescribed by } \\
\text { juniors) }\end{array}$ \\
\hline $\begin{array}{l}\text { Stubbs et al. } \\
\text { (2006) [18] }\end{array}$ & $\begin{array}{l}\text { Retrospective } \\
\text { observational }\end{array}$ & $\begin{array}{l}\text { Setting: eight acute or mental } \\
\text { health trust centres and one } \\
\text { independent psychiatric } \\
\text { hospital } \\
\text { Subjects: patients ( } n=\text { not } \\
\text { reported) attending psychiatric } \\
\text { hospitals; consultant and } \\
\text { nonconsultant psychiatrists } \\
\text { ( } n=\text { not reported) }\end{array}$ & $\begin{array}{l}\text { In accordance with Dean's Delphi } \\
\text { study of prescribing errors [32] }\end{array}$ & None & $\begin{array}{l}\text { Nonconsultant staff made } 329 \text { of } \\
\text { the } 523 \text { errors }(63 \%) \text {, } \\
\text { calculated as a rate of } 15 \text { errors } \\
\text { made by junior staff per } 1000 \\
\text { items prescribed by all doctors }\end{array}$ \\
\hline $\begin{array}{l}\text { Shaughnessy } \\
\text { et al. (1991) } \\
\text { [20] }\end{array}$ & $\begin{array}{l}\text { Prospective } \\
\text { observational }\end{array}$ & $\begin{array}{l}\text { Setting: university-affiliated } \\
\text { outpatient family medicine } \\
\text { teaching centre (primary care) } \\
\text { Subjects: patients attending } \\
\text { ( } n=\text { not reported); family } \\
\text { practice residents }(n=20) \text { in } \\
\text { their } 1 \text { st, } 2 \text { nd or } 3 \text { rd year of } \\
\text { training }\end{array}$ & $\begin{array}{l}\text { Major or minor omissions of } \\
\text { critical information, dose or } \\
\text { direction error, failure to meet } \\
\text { legal requirements, } \\
\text { nonprescription product, } \\
\text { unclear quantity, or incomplete } \\
\text { chart }\end{array}$ & $\begin{array}{l}\text { Physician education using } \\
\text { copies of prescriptions } \\
\text { written by study } \\
\text { participants to provide } \\
\text { feedback on } \\
\text { prescription-writing skills }\end{array}$ & $\begin{array}{l}21 \% \text { of charts contained an error } \\
\text { prior to the intervention and } \\
17 \% \text { after the intervention }\end{array}$ \\
\hline $\begin{array}{l}\text { Shaughnessy } \\
\text { and D'Amico } \\
\text { (1994) [21] }\end{array}$ & $\begin{array}{l}\text { Prospective } \\
\text { observational }\end{array}$ & $\begin{array}{l}\text { Setting: community hospital } \\
\text { outpatient family medicine } \\
\text { teaching centre (primary care) } \\
\text { Subjects: patients attending } \\
\text { ( } n=\text { not reported); family } \\
\text { practice residents }(n=12) \text { in } \\
\text { their } 1 \text { st or } 2 \text { nd year of training }\end{array}$ & $\begin{array}{l}\text { Major or minor omissions of } \\
\text { critical information, dose or } \\
\text { direction error, failure to meet } \\
\text { legal requirements, } \\
\text { nonprescription product, } \\
\text { unclear quantity, or incomplete } \\
\text { chart }\end{array}$ & $\begin{array}{l}\text { An educational programme } \\
\text { consisting of evaluation } \\
\text { and feedback of } \\
\text { prescription writing by a } \\
\text { clinical pharmacist }\end{array}$ & $\begin{array}{l}\text { At baseline } 14.4 \% \text { of drug charts } \\
\text { contained an error, with } 6 \% \\
\text { post intervention }\end{array}$ \\
\hline
\end{tabular}


Table 3

Continued

\section{Study Study design Setting and subjects \\ Setting: paediatric hospital \\ Kozer et al. \\ Retrospective emergency department (2002) [22] observationa \\ Subjects: patient ( $n=1532)$ attending department; doctors $(n>80)$ defined as staff and trainees (interns, residents and fellows)}

\section{Error definition}

Wrong dose, wrong route of administration, wrong timing or wrong units

\section{Intervention (if applicable) Main findings}

None

The authors report that junior doctors were 1.5 times more likely to commit an error than senior doctors, this would calculate as 162 errors of the total 271, written in 154 charts (2.1 errors made by junior staff per 1000 charts written by all doctors)

$\begin{array}{lcc}\begin{array}{l}\text { McFadzean } \\ \text { et al. (2003) }\end{array} & \text { Prospective } & \text { Setting: medical admissions unit } \\ \text { [23] } & \text { in a district general hospital } \\ & \text { Subjects: patients admitted as } \\ \text { medical emergencies }(n=120) \text {; } & \text { junior doctors }(n=12) \text { towards } \\ & \text { the end of the preregistration } \\ & \text { house officer year/clinical } \\ & \text { pharmacists }(n=4)\end{array}$

Prescribing errors were defined as None drugs omitted or prescribed in error, errors in dosage or frequency, or known drug allergies not recorded.

Drug chart errors were defined as: illegible hand writing, use of lower case, inappropriate use of drug trade names, abbreviation of micrograms or units, omission of date, prescriber's signature, site for topical preparations, frequency, maximum dose and no reason for 'as required' medicines

Wrong dose, wrong route of Preprinted order form vs. wrong units

regular order form
The total no. of errors made by junior doctors in 60 patients was 110 errors in 39 patients (65\% of patients), equalling an error rate of 1.8 per patient.

$49(82 \%)$ of drug charts written by junior doctors had errors, and 12 (20\%) were illegible
At baseline, 411 orders were written on regular charts. 68 errors were identified from the 411 orders, giving a rate of $16.6 \%$

Post intervention 376 orders on new form contained 37 (9.8\%)

\section{Subjects: patients attending on study days (2157 visits); doctors ( $n=$ not reported) defined as staff and trainees (interns, residents and fellows) \\ Kozer et al. Prospective Setting: paediatric hospital \\ (2005) [24] observational emergency department} errors.

No actual data are reported on junior doctors, although the paper states there was no statistically significant difference between the rates of error

\begin{tabular}{|c|c|c|c|c|c|}
\hline $\begin{array}{l}\text { Kozer et al. } \\
\text { (2006) [25] }\end{array}$ & $\begin{array}{l}\text { Prospective } \\
\text { observational }\end{array}$ & $\begin{array}{l}\text { Setting: tertiary paediatric hospital } \\
\text { emergency department } \\
\text { (secondary care) } \\
\text { Subjects: patients ( } n=2157 \text { ) } \\
\text { attending department; doctors } \\
\text { ( } n=22 \text { ) in the ED (interns, } \\
\text { 1st-4th year residents, fellows) }\end{array}$ & $\begin{array}{l}\text { Wrong dose, wrong route of } \\
\text { administration, wrong timing or } \\
\text { wrong units }\end{array}$ & $\begin{array}{l}\text { A short educational tutorial } \\
\text { aimed at reducing the } \\
\text { incidence of prescribing } \\
\text { errors among trainees }\end{array}$ & $\begin{array}{l}976 \text { drug orders were written by } \\
\text { junior doctors, but only } 899 \\
\text { were included in the study } \\
\text { (unable to identify prescriber). } \\
\text { Of these } 112 \text { errors were } \\
\text { identified, giving a rate of } \\
12.5 \% \\
\text { No difference was seen between } \\
\text { error rates of doctors attending } \\
(12.7 \%) \text { and not attending } \\
(12.4 \%) \text { the tutorial }\end{array}$ \\
\hline $\begin{array}{l}\text { Mandal and } \\
\text { Fraser (2005) } \\
\text { [26] }\end{array}$ & $\begin{array}{l}\text { Prospective } \\
\text { observational }\end{array}$ & $\begin{array}{l}\text { Setting: single ophthalmic } \\
\text { hospital } \\
\text { Subjects: patients ( } n=\text { not } \\
\text { reported) attending either } \\
\text { outpatients, A\&E, day care } \\
\text { or as inpatients; } \\
\text { doctors ( } n=\text { not reported) } \\
\text { working within these areas }\end{array}$ & $\begin{array}{l}\text { Errors of prescription writing } \\
\text { (incorrect patient details, } \\
\text { illegibility, incorrect format, } \\
\text { scripts where prescriber could } \\
\text { not be identified) or } \\
\text { drug-related errors (wrong } \\
\text { dose, frequency, route) }\end{array}$ & None & $\begin{array}{l}\text { Junior doctors were responsible } \\
\text { for } 83 \text { charts with at least one } \\
\text { error, which calculates to } 4.2 \% \\
\text { of all charts }\end{array}$ \\
\hline $\begin{array}{l}\text { Taylor et al. } \\
\text { (2005) [27] }\end{array}$ & $\begin{array}{l}\text { Retrospective } \\
\text { observational }\end{array}$ & $\begin{array}{l}\text { Setting: suburban academic } \\
\text { tertiary care children's hospital } \\
\text { Emergency Department } \\
\text { (secondary care) } \\
\text { Subjects: patients ( } n=\text { not } \\
\text { reported) attending } \\
\text { department; } \\
\text { residents ( } n=49 \text { ) }\end{array}$ & $\begin{array}{l}\text { Major or minor omissions of } \\
\text { critical information, wrong dose } \\
\text { or directions, unclear quantity, } \\
\text { incomplete directions or allergy } \\
\text { error (no documentation of } \\
\text { known allergy or use of a drug } \\
\text { for which an allergy has been } \\
\text { reported) }\end{array}$ & None & $\begin{array}{l}212(59 \%) \text { charts out of } 358, \\
\text { contained a total of } 311 \text { errors } \\
\text { per chart. This calculates as } \\
1.47 \text { errors per affected patient }\end{array}$ \\
\hline
\end{tabular}


Table 3

Continued

\begin{tabular}{|c|c|c|c|c|c|}
\hline Study & Study design & Setting and subjects & Error definition & Intervention (if applicable) & Main findings \\
\hline $\begin{array}{l}\text { Webbe et al. } \\
\text { (2007) [28] }\end{array}$ & $\begin{array}{l}\text { Prospective } \\
\text { observational }\end{array}$ & $\begin{array}{l}\text { Setting: } 800 \text {-bed associate } \\
\text { teaching hospital with } \\
\text { catchment population of } \\
320000 \text {. } \\
\text { Subjects: patients ( } n=\text { not } \\
\text { reported) on four wards } \\
\text { admitting general medical } \\
\text { emergencies; } \\
\text { junior doctors on medical wards } \\
(n=13)\end{array}$ & $\begin{array}{l}\text { Transcription errors; failure } \\
\text { to take into account } \\
\text { pharmaceutical issues } \\
\text { (intravenous drug } \\
\text { incompatibilities, drug } \\
\text { interactions, contraindications, } \\
\text { lack of monitoring of drug or } \\
\text { patient parameters); failure to } \\
\text { communicate essential } \\
\text { information (such as omissions } \\
\text { in medication history taking); } \\
\text { use of drugs or doses } \\
\text { inappropriate for the patient }\end{array}$ & $\begin{array}{l}\text { A clinical teaching pharmacist } \\
\text { programme to improve } \\
\text { prescribing skills among } \\
\text { newly qualified (PRHOs) }\end{array}$ & $\begin{array}{l}\text { Pre-intervention: } \\
\text { control group: } 31.8 \% \text { (34/107); } \\
\text { intervention group: } 20 \% \\
\text { (39/195). } \\
\text { Post intervention: } \\
\text { control group: } 25.8 \%(41 / 159) ; \\
\text { intervention group: } 12.5 \% \\
\text { (12/96). } \\
\text { Average baseline incidence of } \\
\quad \text { errors 24.2\% }\end{array}$ \\
\hline $\begin{array}{l}\text { Dean et al. } \\
\text { (2002) [4] }\end{array}$ & $\begin{array}{l}\text { Prospective } \\
\text { observational }\end{array}$ & $\begin{array}{l}\text { Setting: UK hospital (single } \\
\text { centre) } \\
\text { Subjects: inpatients ( } n=\text { not } \\
\text { reported); } \\
\text { doctors }(n=41) \text { in a 550-bed } \\
\text { teaching hospital }\end{array}$ & $\begin{array}{l}\text { In accordance with Dean's Delphi } \\
\text { study of prescribing errors [32] }\end{array}$ & None & $\begin{array}{l}\text { Total of } 88 \text { errors made. Doctor } \\
\text { identifiable in only } 50 \text {. Of these } \\
44 \text { errors were studied, } 40 \\
\text { were made by junior doctors } \\
(91 \%)\end{array}$ \\
\hline $\begin{array}{l}\text { Larson et al. } \\
\text { (2004) [30] }\end{array}$ & $\begin{array}{l}\text { Retrospective } \\
\text { observational }\end{array}$ & $\begin{array}{l}\text { Setting: } 360 \text {-bed } \\
\text { community-based teaching } \\
\text { hospital } \\
\text { Subjects: patients not specified } \\
(n=\text { not reported); surgical } \\
\text { house staff (PGY1-5; } n=22)\end{array}$ & $\begin{array}{l}\text { Orders contraindicated due to } \\
\text { allergy, duplicate or incomplete } \\
\text { orders, failure to take into } \\
\text { account laboratory results or } \\
\text { patient weight (where } \\
\text { necessary), inappropriate dose, } \\
\text { route or frequency, or wrong } \\
\text { patient }\end{array}$ & None & $\begin{array}{l}\text { A total of } 75 \text { errors were made } \\
\text { by } 22 \text { surgical residents }\end{array}$ \\
\hline
\end{tabular}

Kozer [22] studied the incidence and type of prescribing errors in a Canadian paediatric hospital emergency department. Prescribing errors were identified in 154 out of 1532 charts $(10.1 \%)$, or of 766 charts where medicines were prescribed $(20.1 \%)$. There were 271 different prescribing errors in the 154 charts, calculated as 1.8 errors per chart. Three hundred and forty-six patients were treated by juniors, but no indication is given of the number of errors in these patients. However, the authors do report that junior doctors were 1.5 times more likely to commit an error than senior doctors, which would calculate as 162 errors of the total 271 , written in 154 charts (2.1 errors made by junior staff per 1000 charts written by all doctors).

McFadzean [23] compared the accuracy of drug history and chart writing between 12 junior doctors and four clini- cal pharmacists in a UK medical admissions unit. Prescribing errors and drug chart errors were defined as separate categories. Prescribing errors were defined as drugs omitted or prescribed in error, errors in dosage or frequency, or known drug allergies not recorded. Drug chart errors were defined as: illegible hand writing, lower case, inappropriate use of trade names, abbreviation of micrograms or units, or omission of date, signature, site for topical preparations, frequency, maximum dose and reason for 'as required' medicines. Of the 60 patients seen by the junior doctors, there were prescribing errors identified for 39 patients. Forty-nine patients had drug chart errors identified (82\%). There were 110 errors in total. The authors report a rate of 1.8 errors made by juniors per patient chart. 
In a further study in a Canadian paediatric hospital emergency department, Kozer [24] tested an intervention to see if a structured drug order sheet reduced the incidence of medication errors. At baseline, 68 errors were identified from the 411 charts, giving a rate of $16.6 \%$. No actual data are reported on junior doctors, although the paper states there was no statistically significant difference between the rates of error committed by junior and senior doctors.

Kozer [25] determined whether a short tutorial on prescribing (based on previous study results) reduced the incidence of prescribing errors by 22 junior doctors, comparing those who did and did not attend (in the same setting as before). As there was no difference in performance, the results are combined here. There were 976 drug charts written by junior doctors. In 899 the prescriber was identifiable, and 112 drug charts containing at least one error were identified, calculated as $12.5 \%$ of charts.

Mandal [26] studied the number of prescribing errors, where they occurred most commonly and who was most likely to have committed them made over a 4-week period in a single UK ophthalmic hospital. Of the 1952 drug charts screened, 144 (7\%) had an error in prescription writing (defined as incorrect patient details or illegibility). The prescriber could be identified in 126 of the 144 charts with an error. Of the charts where the prescriber could be identified, 68 (54\%) were attributable to junior doctors. Of the remaining 1808 charts with no writing error, 15 (1\%) contained a drug error (defined as incorrect dose, timing or route of administration); all of which were attributable to junior doctors. Junior doctors were therefore responsible for 83 charts with at least one error, which calculates as $4.2 \%$ of all charts.

Taylor [27] determined the frequency, type and severity of prescribing errors in a paediatric emergency department in a US tertiary care teaching hospital. There were 311 errors in 212 charts out of 358 written by juniors, giving a rate of $59 \%$ of charts with errors and 1.5 errors per affected patient.

Webbe [28] studied the effect of a pharmacist intervention for 13 preregistration house officers (PRHOs) in a UK teaching hospital on improving prescribing skills. Baseline errors for all PRHOs were 73 errors in 302 charts, giving a rate of $24 \%$.

\section{Studies reporting total errors}

Dean [4] defined prescribing errors and determined their incidence and causes using Human Error Theory in a UK hospital. There were 88 potentially serious errors in a 2-month period. In 50 of these the prescriber was identified (46 individuals). Forty-four errors were investigated, with junior doctors responsible for 40 errors (91\%).

Haw [29] studied the nature, frequency and severity of prescribing errors in a UK psychiatric hospital over 1 month. During the month long data study period, 311 prescribing errors in 260 prescribed items were identified.
The number of prescription items or charts written in that study period are not reported. Instead, the authors report a separate estimate was made of the error rate $(2.2 \%$ of charts written by all staff). During the study period, junior doctors were noted to make 120 errors and consultants 172 errors from the 311 identified (it appears that 19 errors were made where the prescriber was not identified). No calculations of error rate could be made from this study.

Larson [30] assessed the relative frequency of different types of error made by 22 residents in a surgical training programme in a community-based teaching hospital in the USA. Seventy-five errors were identified over a 2-year period.

\section{Studies reporting percentage of errors by juniors}

Galanter [31] evaluated the introduction of an automated Decision Support Alerts in a computerized physician order entry system in a US teaching hospital, focusing on reducing contraindicated drugs in renal insufficiency. Alerts were taken as a proxy measure for errors. A reduction in the likelihood of the patient receiving a dose of a contraindicated medication was demonstrated; medical house staff (junior doctors) were reported to have prescribed $70 \%$ of all orders that generated an alert.

\section{Discussion}

Twenty-four studies meeting the inclusion criteria were identified and data abstracted. The range of error rates was 2-514 per 1000 items prescribed and $4.2-82 \%$ of charts reviewed.

This review reports the wide ranges of error rates seen, which cannot be compared due to differences in methodology and error definitions used. Even within similar methods and definitions, large differences may be seen by, for example, using errors made by juniors of all items or patients, or only errors in patients that junior doctors have treated. Moreover, settings and grades of junior doctors vary across studies, and comparisons with more senior staff are not made consistently.

Without consistent methods, reporting units and error definitions it is hard to draw meaningful conclusions. Coherent arguments can be made in favour of error per prescribed item or per patient/chart written as the ideal outcome. These two pieces of information tell researchers, clinicians and users of the data working towards improving patient safety two different things. First, how common is an error each time a prescription item is ordered, and second, what is the risk to each individual patient. As such, both pieces of data should be collected and reported. Many studies reported only one rate, and methods of calculating that rate were not consistent or always explicit. No study considered rates of error per individual prescriber, or clustering effects. Such data would provide another useful 
stream of information about the causes of prescribing errors, which would inform future interventions. For example, if all prescribers make similar errors at similar rates, an intervention should be targeted to all prescribers. If, however, most errors are made by a few prescribers, a different approach would be needed. To date, scant literature has been identified that explores the process leading to errors.

Agreement on a standard definition is urgently required, as demonstrated by the wide range of definitions used in the studies we reviewed. Thirty-five separate criteria were noted from studies, which used varying combinations of these. A strong contender for the 'ideal' definition is Dean's Delphi derived definition [32], which represents the result of an expert consensus (doctors, pharmacists and nurses). This definition has the benefit of describing both elements of prescription writing and of decision making, but where all elements are a possible danger to patients.

Other possible reasons for variation in the error range should be considered. No effect of a change in error rates with time of study was seen, suggesting that there has been no rationalizing of methodology over time or improvement in prescribing competence. Nor was a geographical effect observed, suggesting neither a consistency of methodology nor of error rates in particular countries. In fact, the review reveals a very widespread problem that does not appear to be affected by different training models, healthcare systems or infrastructure and automation.

This study was limited by the above methodological differences, and the difficulties in obtaining all papers identified by the search. A number of potentially relevant studies were also excluded due to lack of reporting of the grade of prescriber. It is unlikely given the wide range of error rates and the difficulties in comparison that further data would have added to the overall interpretation of this review.

Interventions aimed at improving prescribing and reducing errors are a vital component in improving patient safety. The size of the problem that should be addressed is no clearer following this review. Baseline data should be collected as a priority in order to evaluate potential interventions. Two directions for future research are suggested that will provide the information needed. First, future research in prescribing error rates should be well constructed and generalizable using standard definitions and methodology. A well-conducted study of prescribing errors by junior doctors is urgently needed. Second, further in-depth research into the reasons for errors using Human Error Theory is required, building on the work done by Dean et al. [4]. Although attention should continue to be focused on systems factors, individual factors should not be discounted. Future research should concentrate on providing the theoretical foundations prior to developing and validating actual interventions.

\section{Competing interests}

None to declare.

This study was supported by a grant from NHS Grampian Endowments. The authors thank Pam Royle for undertaking the majority of the database searching.

\section{Appendix 1}

Example search using MEDLINE database.

Ovid MEDLINE(R) 1950 to July week 32007 was searched with the following terms:

1. (prescribing adj4 error\$).tw.

2. (prescription adj4 error\$).tw.

3. ((prescription or prescribing) adj4 mistake\$).tw.

4. (drug adj1 error\$).tw.

5. (medication adj error\$).tw.

6. (adverse adj2 drug\$ adj2 event\$).tw.

7. (adverse adj2 drug\$ adj2 reaction\$).tw.

8. (medication adj2 adverse adj2 event\$).tw.

9. exp Prescriptions, Drug/

10. exp Medication Errors/

11. Patient Care/

12. exp Physicians/

13. exp Medical Staff/

14. exp Hospitals/

15. exp Primary Health Care/

16. junior.tw.

17. doctor\$.tw.

18. medical staff.tw.

19. 1 or 2 or 3

20.4 or 5 or 6 or 7 or 8 or 9 or 10

21.11 or 12 or 13 or 14 or 15 or 16 or 17 or 18

22. 20 and 21

23. 19 or 22

24. limit 23 to (english language and years $={ }^{\prime} 1990-2007 '$ )

\section{Appendix 2}

Dean's definition of a prescribing error

Prescribing a drug for a patient for whom, as a result of a co-existing clinical condition, that drug is contraindicated

Prescription of a drug to which the patient has a documented clinically significant allergy

Not taking into account a potentially significant drug interaction

Prescribing a drug in a dose that, according to British National Formulary or data sheet recommendations, is inappropriate for the patient's renal function 
Prescription of a drug in a dose below that recommended for the patient's clinical condition

Prescribing a drug with a narrow therapeutic index, in a dose predicted to give serum levels significantly above the desired therapeutic range

Writing a prescription for a drug with a narrow therapeutic range in a dose predicted to give serum levels significantly below the desired therapeutic range

Not altering the dose following steady-state serum levels significantly outside the therapeutic range

Continuing a drug in the event of a clinically significant adverse drug reaction

Prescribing two drugs for the same indication when only one of the drugs is necessary

Prescribing a drug for which there is no indication for that patient

Prescribing a drug to be given by intravenous infusion in a diluent that is incompatible with the drug prescribed

Prescribing a drug to be infused via an intravenous peripheral line, in a concentration greater than that recommended for peripheral administration

Failure to communicate essential information

Prescribing a drug, dose or route that is not that intended

Writing illegibly

Writing a drug's name using abbreviations or other nonstandard nomenclature

Writing an ambiguous medication order

Prescribing 'one tablet' of a drug that is available in more than one strength of tablet

Omission of the route of administration for a drug that can be given by more than one route

Prescribing a drug to be given by intermittent intravenous infusion, without specifying the duration over which it is to be infused

Omission of the prescriber's signature

On admission to hospital, unintentionally not prescribing a drug that the patient was taking prior to their admission

Continuing a GP's prescribing error when writing a patient's drug chart on admission to hospital

Transcribing a medication order incorrectly when rewriting a patient's drug chart Writing 'milligrams' when 'micrograms' was intended
Writing a prescription for discharge medication that unintentionally deviates from the medication prescribed on the inpatient drug chart

On admission to hospital, writing a medication order that unintentionally deviates from the patient's pre-admission prescription

Prescribing a drug in a dose above the maximum dose recommended in the British National Formulary or data sheet

Misspelling a drug name

Prescribing a dose that cannot readily be administered using the dosage forms available

Prescribing a dose regime (dose/frequency) that is not that recommended for the formulation prescribed

Continuing a prescription for a longer duration than necessary

Prescribing a drug that should be given at specific times in relation to meals without specifying this information on the prescription

Unintentionally not prescribing a drug for a clinical condition for which medication is indicated

The criteria in italics may be errors depending on the circumstances.

\section{REFERENCES}

1 National Patient Safety Agency. Patient safety incident reports in the NHS: National Reporting and Learning System Data Summary. Issue 7. Available at http://www.npsa.nhs.uk/ patientsafety/patient-safety-incident-data/quarterly-datareports (last accessed 19 May 2008).

2 Barber N, Rawlins M, Franklin BD. Reducing prescribing error: competence, control, and culture. Qual Saf Health Care 2003; 12:29-32.

3 Reason J. Human Error. Cambridge: University of Cambridge, 1990.

4 Dean B, Schachter M, Vincent C, Barber N. Causes of prescribing errors in hospital inpatients: a prospective study. Lancet 2002; 359: 1373-8.

5 Franklin BD, Vincent C, Schachter M, Barber N. The incidence of prescribing errors in hospital inpatients: an overview of the research methods. Drug Saf 2005; 28: 891-900.

6 Scottish Intercollegiate Guidelines Network. Available at http://www.sign.ac.uk (last accessed 21 October 2007).

7 Critical Appraisal Skills Programme. Available at http://www.phru.nhs.uk/Pages/PHD/resources.htm (last accessed 21 October 2007). 
8 Effective Public Health Practice Project. Quality Assessment Tool for Quantitative Studies 2003 (Effective Practice, Informatics and Quality Improvement). Available at: http://www.myhamilton.ca/NR/rdonylres/6B3670AC8134-4F76-A64C-9C39DBC0F768/0/QATool.pdf

9 Lesar TS, Briceland LL, Delcoure K, Parmalee JC, Masta-Gornic V, Pohl H. Medication prescribing errors in a teaching hospital. JAMA 1990; 263:2329-34.

10 Bordun LA, Butt W. Drug errors in intensive care. J Paediatr Child Health 1992; 28: 309-11.

11 Ho L, Brown GR, Millin B. Characterization of errors detected during central order review. Can J Hosp Pharm 1992; 45: 193-7.

12 Howell RR, Jones KW. Prescription-writing errors and markers: the value of knowing the diagnosis. Fam Med 1993; 25: 104-6.

13 Bizovi KE, Beckley BE, McDade MC, Adams AL, Lowe RA, Zechnich AD, Hedges JR. The effect of computer-assisted prescription writing on emergency department prescription errors. Acad Emerg Med 2002; 9: 1168-75.

14 Dean B, Schachter M, Vincent C, Barber N. Prescribing errors in hospital inpatients: their incidence and clinical significance. Qual Saf Health Care 2002; 11:340-4.

15 Fijn R, van den Bemt PM, Chow M, De Blaey CJ, de Jong-van den Berg LT, Brouwers JR. Hospital prescribing errors: epidemiological assessment of predictors. $\mathrm{Br} \mathrm{J}$ Clin Pharmacol 2002; 53: 326-31.

16 Anton C, Nightingale PG, Adu D, Lipkin G, Ferner RE. Improving prescribing using a rule based prescribing system. Qual Saf Health Care 2004; 13: 186-90.

17 Hendey GW, Barth BE, Soliz T. Overnight and postcall errors in medication orders. Acad Emerg Med 2005; 12: 629-34.

18 Stubbs J, Haw C, Taylor D. Prescription errors in psychiatry a multi-centre study. J Psychopharmacol 2006; 20: 553-61.

19 Walsh KE, Adams WG, Bauchner H, Vinci RJ, Chessare JB, Cooper MR, Hebert PM, Schainker EG, Landrigan CP. Medication errors related to computerized order entry for children. Pediatrics 2006; 118: 1872-9.
20 Shaughnessy AF, D'Amico F, Nickel RO. Improving prescription-writing skills in a family practice residency. DICP 1991; 25: 17-21.

21 Shaughnessy AF, D'Amico F. Long-term experience with a program to improve prescription-writing skills. Fam Med 1994; 26: 168-71.

22 Kozer E, Scolnik D, Macpherson A, Keays T, Shi K, Luk T, Koren G. Variables associated with medication errors in pediatric emergency medicine. Pediatrics 2002; 110: 737-42.

23 McFadzean E, Isles C, Moffat J, Norrie J, Stewart D. Is there a role for a prescribing pharmacist in preventing prescribing errors in a medical admission unit? Pharm J 2003; 270: 896-9.

24 Kozer E, Scolnik D, Macpherson A, Rauchwerger D, Koren G. Using a preprinted order sheet to reduce prescription errors in a pediatric emergency department: a randomized, controlled trial. Pediatrics 2005; 116: 1299-302.

25 Kozer E, Scolnik D, Macpherson A, Rauchwerger D, Koren G. The effect of a short tutorial on the incidence of prescribing errors in pediatric emergency care. Can J Clin Pharmacol 2006; 13: e285-91.

26 Mandal K, Fraser SG. The incidence of prescribing errors in an eye hospital. BMC Ophthalmol 2005; 5: 4 .

27 Taylor BL, Selbst SM, Shah AE. Prescription writing errors in the pediatric emergency department. Pediatr Emerg Care 2005; 21: 822-7.

28 Webbe D, Dhillon S, Roberts CM. Improving junior doctor prescribing - the positive impact of a pharmacist intervention. Pharm J 2007; 278: 136-8.

29 Haw C, Stubbs J. Prescribing errors at a psychiatric hospital. Pharm Pract 2003; 13: 64-6.

30 Larson KA, Wiggins EF, Goldfarb MA. Reducing medication errors in a surgical residency training program. Am Surg 2004; 70: 467-71.

31 Galanter WL, Didomenico RJ, Polikaitis A. A trial of automated decision support alerts for contraindicated medications using computerized physician order entry. J Am Med Inform Assoc 2005; 12: 269-74.

32 Dean B, Barber N, Schachter M. What is a prescribing error? Qual Health Care 2000; 9: 232-7. 